\title{
A facile method to modify bentonite nanoclay with silane
}

\author{
Sujani B. Y. Abeywardena ${ }^{1,3} \cdot$ Srimala Perera $^{2}$ (1) K. M. Nalin de Silva ${ }^{3,4}$ • \\ Nadeeka P. Tissera ${ }^{3}$
}

Received: 18 March 2017/ Accepted: 17 July 2017 /Published online: 21 July 2017

(c) The Author(s) 2017. This article is an open access publication

\begin{abstract}
Immobilization of smectite clay onto a desirable surface has received much attention, since its nanospace can be utilized for many applications in material science. Here, we present an efficient method to functionalize surface of bentonite nanoclay (BNC) through the grafting of 3-aminotriethoxysilane (APTES). Infrared spectroscopy and elemental analysis confirmed the presence of organic chains and amine groups in modified nanoclay. XRD analysis confirmed grafting of APTES on the surface of bentonite nanoclay without intercalation. The accomplishment of the surface modification was quantitatively proved by TGA analysis. Modified BNC can covalently couple with different material surfaces, allowing its nanospace to be utilized for intercalation of cations, bio-molecules, and polymeric materials, to be used in advanced military aerospace, pharmaceuticals, and many other commercial applications.
\end{abstract}

Keywords Bentonite nanoclay $\cdot$ Covalent modification . Nanospace $\cdot$ Silane $\cdot$ Surface $\cdot$ Spectroscopy

Srimala Perera

srimalaperera@gmail.com

1 Department of Chemical and Process Engineering, Faculty of Engineering, University of Moratuwa, Moratuwa, Sri Lanka

2 Division of Polymer, Textile and Chemical Engineering Technology, Institute of Technology, University of Moratuwa, Moratuwa, Sri Lanka

3 Sri Lanka Institute of Nanotechnology (Pvt) Ltd, Nanotechnology and Science Park, Homagama, Sri Lanka

4 Department of Chemistry, University of Colombo, Colombo, Sri Lanka

\section{Introduction}

The space in between two layers of smectite clay is in nanometer scale, which is potentially suitable for the accommodation of a range of guest molecules. This nanospace in smectite clay plays an important role in different fields such as polymer nanocomposites, cation adsorption, intercalation, and exfoliation [1-3]. Smectite clay has also shown the ability to release intercalated molecules as and when required [4]. It would be noteworthy if smectite clay can be grafted on different surfaces, while its nanospace is accommodated for special requirements such as targeted drug delivery, disintegrated agents in drug formation, selective removal of heavy metal contaminants, applications in high-temperature environments, and conducting polymers in wearable electronics. In grafting of clay, chemical bonding is more stable and durable compared to physical bonding.

Silane can be used to graft clay particles onto a range of material surfaces $[5,6]$. The hydroxyl groups in clay provide excellent sites for silane grafting. Hydroxyl groups in broken edges of clay particles play a vital role in grafting compared to hydroxyl groups in basal surfaces of clay [5]. Hydrolyzed silane can readily form siloxane bonds with hydroxyl groups in basal surfaces and "broken" edges of swelling clay minerals such as montmorillonite and bentonite. In unrestrained conditions, silane can also be physically adsorbed or intercalated with clay. Some X-ray powder diffraction (XRD) data in the literature show an expansion of nanospace in clay due to intercalation of silane $[7,8]$. Silane is very reactive with moisture in the air. When silane is exposed to moisture, hydrolysis occurs rapidly, forming self-condensed, water insoluble resinous oligomer, and polymer structures. This has led to conduct silylation in anhydrous organic solvents in inert gas 
environments. The rates of both silane hydrolysis and condensation are influenced by variations in $\mathrm{pH}$ levels. However, the optimum $\mathrm{pH}$ for hydrolysis is not optimum for condensation. Finding the best balance between hydrolysis and condensation is one of the keys to the successful grafting of silane.

In a carefully controlled environment, silane-grafted clay compounds contain special functional groups such as $-\mathrm{NH}_{2}$ and $-\mathrm{SH}$, which can be covalently bonded with desirable surfaces [2]. Here, we report a simple one-pot reaction of a silane-grafted $\mathrm{BNC}$ compound, which contains $-\mathrm{NH}_{2}$ for further immobilization on various surfaces. In this study, surface characterization, expansion of nanospace of BNC, and bond formation between APTES and BNC were studied.

\section{Materials and methods}

A dispersion of BNC was obtained by dissolving $4 \mathrm{~g}$ of nanoclay, hydrophilic bentonite nanoclay (Aldrich), in $100 \mathrm{ml}$ of deionized water. Particles of BNC, $100 \mathrm{~nm}$ in size, were obtained by ball milling (FRITSCH PULVERISETTE 7 premium line grinder). $2 \mathrm{mmol} \mathrm{dm}^{-3}$ of APTES solution was prepared by controlled dropwise addition of APTES (Aldrich 99\%) to deionized water. A precipitation was obtained by $1 \mathrm{ml} / \mathrm{min}$ drop rate addition of BNC to APTES solution without agitation in an ambient environment. The slurry was immediately centrifuged (Sigma 3-18) at $9000 \mathrm{rpm}$ for $15 \mathrm{~min}$ and washed five times with deionized water. The collected solid was finally dried at $110{ }^{\circ} \mathrm{C}$ for $6 \mathrm{~h}$. Modified and non-modified samples were characterized by Fourier transform infrared spectrophotometry (FTIR), XRD, thermogravimetry (TG), and scanning electron microscopy (SEM). XRD profiles were recorded on a Bruker PW 1050 diffractometer using $\mathrm{Cu}-K \alpha$ radiation $(\lambda=0.154 \mathrm{~nm})$, over a $2 \theta$ range of $3^{\circ}-$ $70^{\circ}$, step size of 0.01 , and a step time of 2 s. FTIR spectra were obtained on a Bruker Vertex 80 coupled with Ram-FT module (RAM II, DTGS detector, $4000-400 \mathrm{~cm}^{-1}$ spectral range, resolution of $4 \mathrm{~cm}^{-1}$, OPUS software). TGA was carried out on an SDT Q600 thermoanalyser (TA Instrument, sample mass $\sim 10 \mathrm{mg}$; heating rate $10^{\circ} \mathrm{C} / \mathrm{min}$; nitrogen flow). SEM and energy dispersive X-ray spectroscopy (EDX) analysis were recorded using HITACHI SU6600 microscope coupled with EDX detector.

\section{Results and discussion}

The above characterization methods were performed to investigate the expansion of nanospace, covalent bonding, and surface morphology of APTES-grafted BNC.
XRD spectra a (BNC, Fig. 1a) and b (APTES-modified BNC, Fig. 1b) show no significant shift in the typical peak of (001) plane, which corresponds to the basal spacing of clay [9]. Spectrum exhibits a peak at $2 \theta=7.8^{\circ}$ corresponding to the (001) plane, indicating the basal spacing of $1.47 \mathrm{~nm}$. The immediately centrifuged and dried slurry (spectrum b) exhibits the basal spacing of $1.59 \mathrm{~nm}$. When reaction time of the same was increased to $24 \mathrm{~h}$ with constant agitation, the basal spacing increased to $2.01 \mathrm{~nm}$ (spectrum c) showing a significant expansion of the nanospace of BNC. This shows intercalation of APTES in nanospace of BNC. However, the slight shift in the peak of (001) plane in spectrum b confirms grafting of APTES on broken edges of BNC. Furthermore, the clear difference in the peaks corresponding to (001) plane in spectra $b$ and $c$ is due to the difference in reaction conditions in the methodology.

Bentonite clay in nanoscale increases the hydroxyl groups on the broken edges of its surface, facilitating a rapid silylation. Dropwise addition of APTES to deionized water reduces the ability to polymerize the resulted uncondensed aminopropyl-functional silanetriol monomer. Hence, these uncondensed silanols lead to react with hydroxyl groups on the surface of BNC, forming a stable Si-O-Si bond on drying. When a short time is given for the reaction in low concentration of APTES, grafting mainly occurs on the broken edges of BNC. When stirring and increasing the reaction time, grafting reaction occurs not only on the edge but also on the interlayer nanospace [10].

Furthermore, delayering of clay can occur under low $\mathrm{pH}$ levels. Adjustment of $\mathrm{pH}$ was unnecessary, since the alkaline medium created by APTES catalyzed the silane-

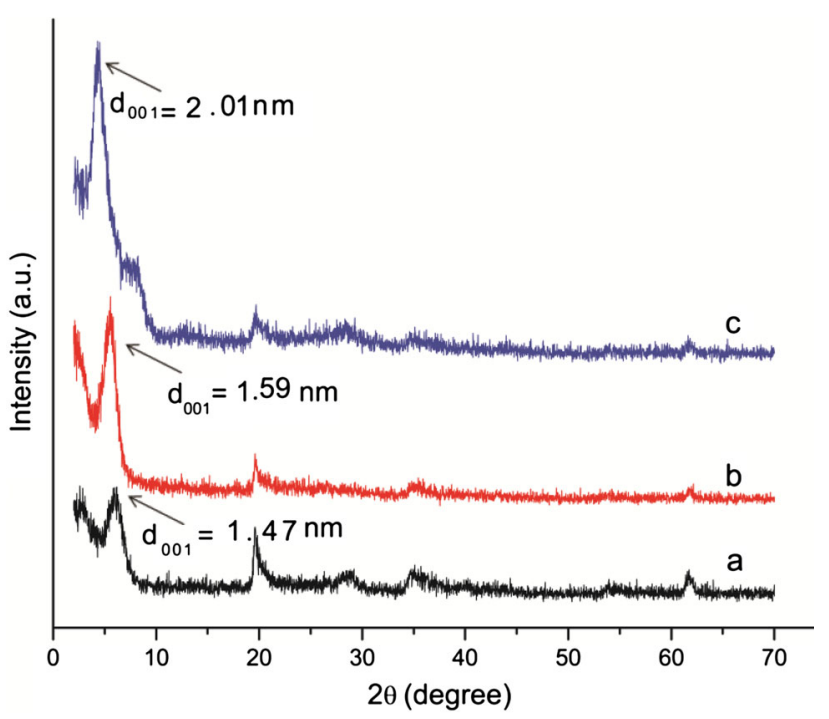

Fig. 1 XRD diffraction patterns of $a$ BNC, $b$ APTES-modified BNC, and $c$ APTES-intercalated BNC 


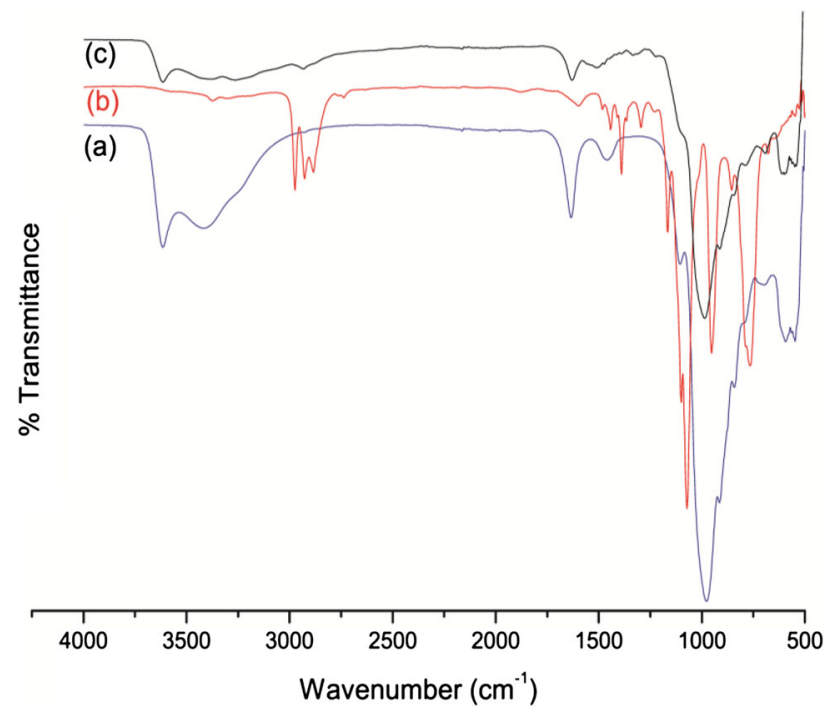

Fig. 2 FTIR spectra of $a$ BNC, $b$ APTES, and $c$ APTES-modified $\mathrm{BNC}$

grafting process, which was an added advantage in silylation of BNC with APTES.

FTIR spectra (Fig. 2) confirm the formation of bonds between APTES and BNC by the presence of characteristic absorption bands relevant to bonding of APTES.

Successfully grafted APTES on BNC can be confirmed by a group of absorption bands in $3000-2800 \mathrm{~cm}^{-1}$ in spectrum (c) (Fig. 2). This absorption is attributed to the valence vibration $v_{\mathrm{s} \text {,as }}(\mathrm{CH})$ of propyl chain in APTES. Its presence is also confirmed by a group of bands of weak intensity in $1490-1300 \mathrm{~cm}^{-1}$ region. Spectra (a) and (c) present the same set of absorption bands relevant to inorganic structure of clay [11]. Since modified and nonmodified BNC contain water, there is a characteristic absorption band of deformation vibrations of $\mathrm{H}_{2} \mathrm{O}$ molecules at $1630 \mathrm{~cm}^{-1}$ and a broad intense absorption band above $3420 \mathrm{~cm}^{-1}$ due to vibrations of $\mathrm{OH}^{-}$groups in water molecules participating in the formation of hydrogen bonds. In spectra (a) and (c), the strong broad band in $1100-900 \mathrm{~cm}^{-1}$ is attributed to valence vibration of $\mathrm{Si}-\mathrm{O}$ bonds in BNC. Moreover, peaks at 3300, 700, and $1340 \mathrm{~cm}^{-1}$ correspond to the $\mathrm{N}-\mathrm{H}$ stretching, C-H-bending vibrations, and $\mathrm{Si}-\mathrm{C}$ stretching, respectively. These peaks support the covalent bonding of APTES onto nanoclay. In spectrum (c), intensive bands in approximately $1200-1100 \mathrm{~cm}^{-1}$ represent the asymmetric stretching of siloxane groups $(\mathrm{Si}-\mathrm{O}-\mathrm{Si})$. These bands correspond to a combination of $\mathrm{Si}-\mathrm{O}-\mathrm{Si}$ vibrational modes including those of bonds formed between the silane and clay surface and cross-linking between silane molecules on the surface [10].

EDX analysis in Fig. 3a shows the presence of significant amount of carbon and nitrogen in APTES-modified BNC, while the scanning electron microphotograph in Fig. $3 b$ shows the surface morphology of APTES-modified BNC. These figures confirm that APTES has successfully grafted on broken edges of BNC.

Thermogravimetric analysis was used to determine the chemically grafted silane on BNC. The thermograms together with derivative thermograms (DTG) for BNC and APTES-modified BNC are shown in Fig. 4. DTG curve in graph (b) shows weight losses occurring at two distinct temperatures. The first weight loss $(14.96 \%)$ occurring at

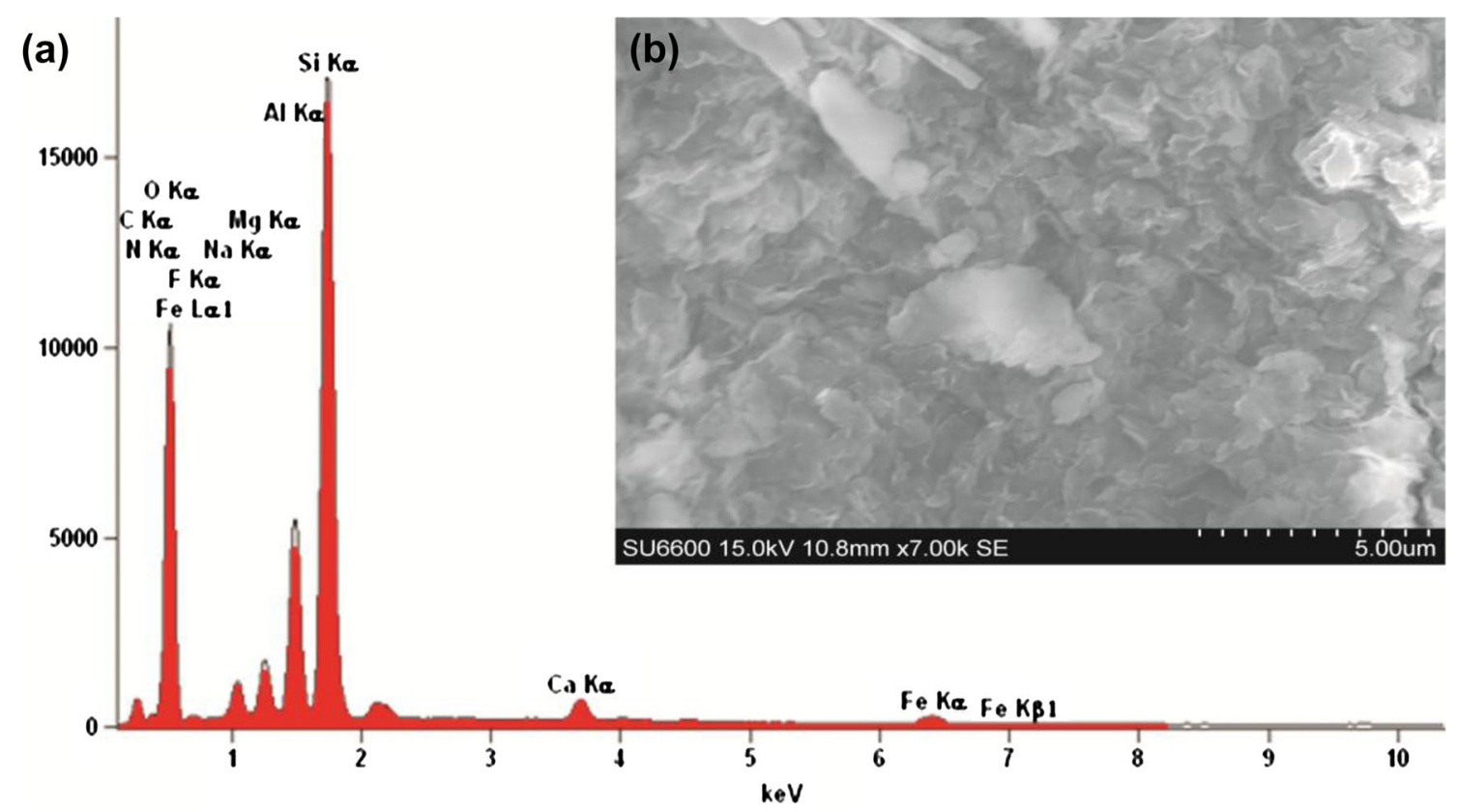

Fig. 3 a SEM-EDX analysis of APTES-modified BNC. b SEM image of APTES-modified BNC 
Fig. 4 Thermograms (in black) and derivative thermograms (in blue) for a APTES, b BNC, and c APTES-grafted BNC
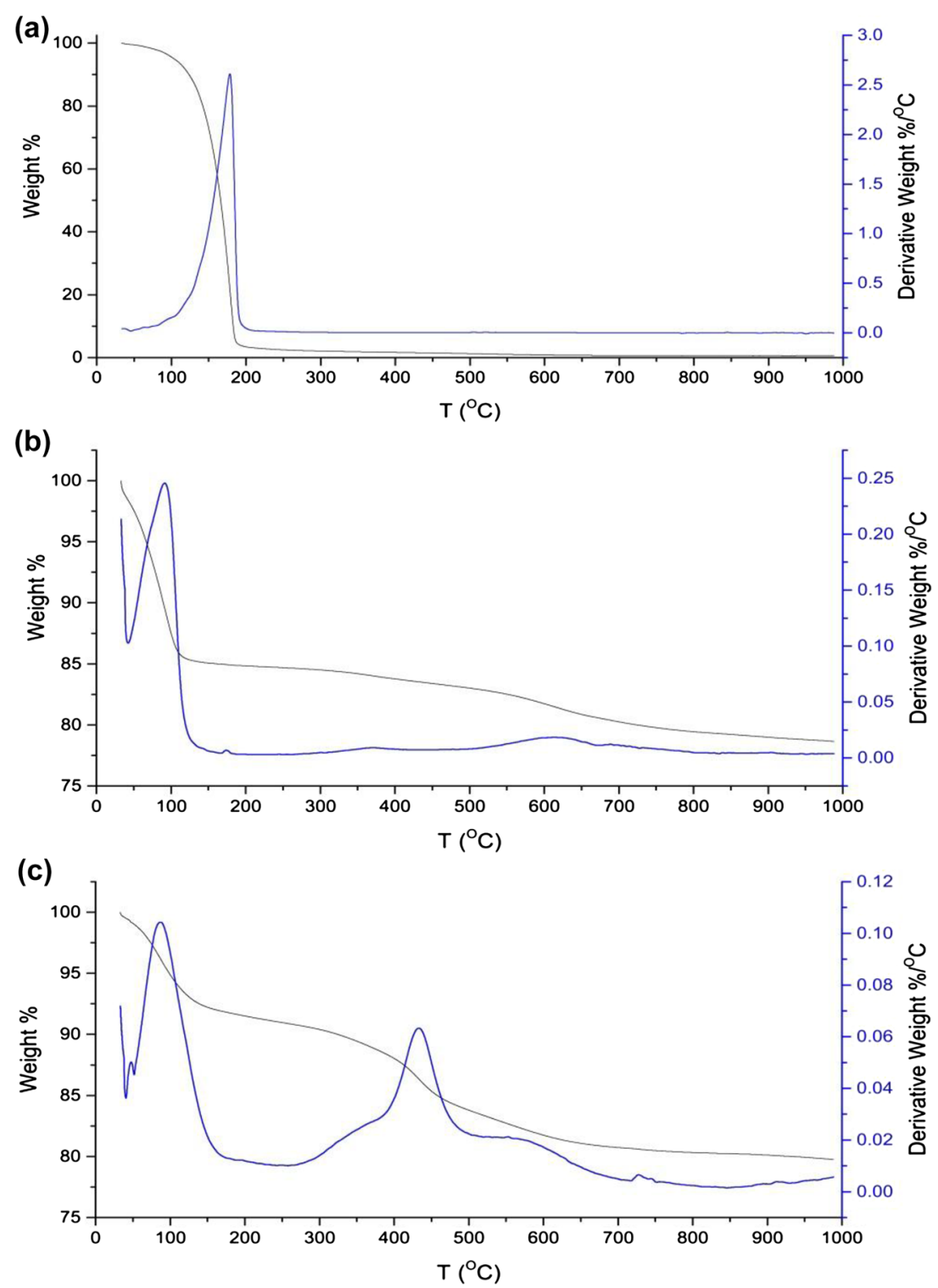

$100{ }^{\circ} \mathrm{C}$ was attributed to the loss of physically adsorbed water, while the second weight loss $(4.17 \%)$ occurring at $645{ }^{\circ} \mathrm{C}$ was attributed to the dehydroxylation of clay [11]. The weight loss $(1.80 \%)$ observed in the range $200-500{ }^{\circ} \mathrm{C}$ was attributed to the dehydroxylation of structural $\mathrm{OH}$ groups in clay [11]. After the grafting of APTES, graph (c) shows an initial weight loss $(8.56 \%)$ taking place up to $150{ }^{\circ} \mathrm{C}$. This can be attributed to the removal of adsorbed surface water. Compared to graph (b), this lesser water content in APTES-grafted BNC indicates that APTES has grafted on BNC. The weight loss observed in the range $200-550{ }^{\circ} \mathrm{C}$ is mainly attributed to the thermal decomposition of organic molecules [12]. The weight loss $(8.49 \%)$ observed in the range $400-575{ }^{\circ} \mathrm{C}$ corresponds to chemically bonded APTES on broken edges of BNC [5]. As in unmodified BNC, the weight loss $(2.92 \%)$ observed in the range $600-800{ }^{\circ} \mathrm{C}$ is due to the dehydroxylation of clay. The final weight of APTES-modified nanoclay is greater than unmodified nanoclay due to organic and inorganic Si contents in APTES.

\section{Conclusions}

APTES-grafted BNC has been obtained by a rapid one-pot reaction under mild conditions. XRD data confirmed grafting APTES on broken edges of BNC, without intercalation. FTIR and TGA confirmed covalent modification of APTES on BNC. Furthermore, SEM-EDX investigation proved the presence of elements in APTES-grafted BNC. This appears as a convenient green modification route to produce APTES-grafted BNC, facilitating several 
applications such as nanoclay vehicles for targeted drug delivery, selective removal of heavy metal contaminants, and intercalation of conducting polymers in wearable electronics.

Acknowledgements This work was financially supported by a shortterm research grant-Institute of Technology, University of Moratuwa, Sri Lanka, 2016. We would also like to thank Sri Lanka Institute of Nanotechnology (Pvt) Ltd (SLINTEC) for providing facilities for the research.

Open Access This article is distributed under the terms of the Creative Commons Attribution 4.0 International License (http://crea tivecommons.org/licenses/by/4.0/), which permits unrestricted use, distribution, and reproduction in any medium, provided you give appropriate credit to the original author(s) and the source, provide a link to the Creative Commons license, and indicate if changes were made.

\section{References}

1. Liu, P.: Polymer modified clay minerals. Appl. Clay Sci. 38, 64-76 (2007)

2. Bergaya, F., Lagaly, G.: Surface modification of clay minerals. Appl. Clay Sci. 19, 1-3 (2001)

3. Cheraghian, G., Nezad, S.S.K., Kamari, M., Hemmati, M., Masihi, M., Bazgir, S.: Adsorption polymer on reservoir rock and role of the nanoparticles, clay and $\mathrm{SiO}_{2}$. Int. Nano Lett. 4, 114 (2014)
4. He, H., Tao, Q., Zhu, J., Yuan, P., Shen, W., Yang, S.: Silylation of clay mineral surfaces. Appl. Clay Sci. 71, 15-20 (2013)

5. Shanmugharaj, A.M., Rhee, K.Y., Ryu, S.H.: Influence of dispersing medium on grafting of aminopropyltriethoxysilane in swelling clay materials. J. Colloid Interface Sci. 298, 854-859 (2006)

6. Ahangaran, F., Haassanzadeh, A., Nouri, S.: Surface modification of $\mathrm{Fe}_{3} \mathrm{O}_{4} @ \mathrm{SiO}_{2}$ microsphere by silane coupling agent. Int. Nano Lett. 3, 23 (2013)

7. Daniel, L.M., Frost, R.L., Zhu, H.Y.: Edge-modification of laponite with dimethyl-octylmethoxysilane. J. Colloid Interface Sci. 321, 302-309 (2008)

8. Herrera, N.N., Letoffe, J.M., Putaux, J.L., David, L., BourgeatLami, E.: Aqueous dispersions of silane-functionalized laponite clay platelets. A first step towards the elaboration of water-based polymer/clay nanocomposites. Langmuir 20, 1564-1571 (2004)

9. Guimaraes, A.M.F., Ciminelli, V.S.T., Vasconcelos, W.L.: Surface modification of synthetic clay aimed at biomolecule adsorption: synthesis and characterization. Mater. Res. 10, 37-41 (2007)

10. He, W., Yao, Y., He, M., Kai, Z., Long, L., Zhang, M., Qin, S., $\mathrm{Yu}, \mathrm{J}$. : Influence of reaction conditions on the grafting pattern of 3-glycidoxypropyl trimethoxysilane on montmorillonite. Bull. Korean Chem. Soc. 34, 113-116 (2013)

11. He, H., Frost, L.R., Zhu, J.: Infrared study of HDTMA ${ }^{+}$intercalated montmorillonite. Spectrochim. Acta Part A Mol. Biomol. Spectrosc. 60, 2853-2859 (2004)

12. Xie, W., Gao, Z., Pan, W.P., Hunter, D., Singh, A., Vaia, R.: Thermal degradation chemistry of alkyl quaternary ammonium montmorillonite. Chem. Mater. 13, 2979-2990 (2001) 